\title{
QoS Aware Cloud Based Routing Protocol for Security Improvement of Hybrid Wireless Network
}

\author{
Uma Khemchand Thakur*, Chandrashekhar Dethe \\ Department of Information Technology, SGBAU University, Amaravati, India \\ Email address: \\ umapatel21@gmail.com (U. K. Thakur),cgdethe@gmail.com (C. Dethe) \\ ${ }^{*}$ Corresponding author
}

\section{To cite this article:}

Uma Khemchand Thakur, Chandrashekhar Dethe. QoS Aware Cloud Based Routing Protocol for Security Improvement of Hybrid Wireless Network. Machine Learning Research. Vol. 4, No. 1, 2019, pp. 21-26. doi: 10.11648/j.mlr.20190401.14

Received: April 9, 2019; Accepted: May 28, 2019; Published: June 20, 2019

\begin{abstract}
The recent advances and the convergence of micro electro-mechanical systems technology, integrated circuit technologies, microprocessor hardware and Nano-technology, wireless communications, Ad-hoc networking routing protocols, distributed signal processing, and embedded systems have made the concept of Wireless Sensor Networks (WSNs). Sensor network nodes are limited with respect to energy supply, restricted computational capacity and communication bandwidth. Most of the attention, however, has been given to the routing protocols since they might differ depending on the application and network architecture. To prolong the lifetime of the sensor nodes, designing efficient routing protocols is critical. Even though sensor networks are primarily designed for monitoring and reporting events, since they are application dependent, a single routing protocol cannot be efficient for sensor networks across all applications. In this paper, we analyze the design issues of sensor networks and present a classification and comparison of routing protocols. This comparison reveals the important features that need to be taken into consideration while designing and evaluating new routing protocols for sensor networks. A reliable transmission of packet data information, with low latency and high energy-efficiency, is truly essential for wireless sensor networks, employed in delay sensitive industrial control applications. The proper selection of the routing protocol to achieve maximum efficiency is a challenging task, since latency, reliability and energy consumption are inter-related with each other. It is observed that, Quality of Service (QoS) of the network can improve by minimizing delay in packet delivery, and life time of the network, can be extend by using suitable energy efficient routing protocol.
\end{abstract}

Keywords: Wireless Sensor Network, Quality of Service, Routing Protocol

\section{Introduction}

Routing is one of the critical technologies in WSNs. Opposed to traditional ad hoc networks, routing in WSNs is more challenging as a result of their inherent characteristics [1]. Firstly, resources are greatly constrained in terms of power supply, processing capability and transmission bandwidth. Secondly, it is difficult to design a global addressing scheme as Internet Protocol (IP). Furthermore, IP cannot be applied to WSNs, since address updating in a large-scale or dynamic WSN can result in heavy overhead. Thirdly, due to the limited resources, it is hard for routing to cope with unpredictable and frequent topology changes, especially in a mobile environment. Fourthly, data collection by many sensor nodes usually results in a high probability of data redundancy, which must be considered by routing protocols [2]. Fifthly, most applications of WSNs require the only communication scheme of many-to-one, i.e., from multiple sources to one particular sink, rather than multicast or peer to peer. Finally, in time-constrained applications of WSNs, data transmissions should be accomplished within a certain period of time.

Thus, bounded latency for data transmissions must be taken into consideration in this kind of applications. Nevertheless, energy conservation is more important than quality of service (QoS) in most applications in that all sensor nodes are constrained with energy which is directly related to network lifetime. 


\section{Literature Review}

Being a reactive routing protocol AODV makes use of routing that is traditional, one admission per location and series data are widely used to see whether routing suggestions was current also to prevent routing loops. Route Request message to their neighbors to get the path to resort. The foundation node broadcast the RREQ in other words. Destination Sequence Number may be the sequence number that is latest obtained in past times by the foundation for almost any path to the resort and supply Sequence quantity could be the latest series number to be utilized within the path entryway pointing to the way to obtain the path request [3]. If any node from a listing of friends is resorted or understands the path to the location, it may send RREP information to the origin. AODV makes use of $<$ RREQ, RREP $>$ pair to get the route. It can help both in unicasting and multicasting. The RREQ message contains the supply and resort target, lifetime of content, sequence variety of supply and resort and request ID as distinctive identification [3].

Destination-Sequenced Distance-Vector (DSDV) Routing Algorithm is founded on the classical Bellman Ford Routing Algorithm with some improvements. So, the modification is that it is both time-driven and event-driven. The stations sporadically transfer their particular routing tables for their neighbors that are immediate. A station furthermore transmits its routing desk in cases where a change that is significant took place the table through the last upgrade sent. The routing table revisions may be submitted two ways: - a "full dump" or perhaps an update that is incremental. Once the network is fairly stable, incremental revisions include sent to prevent traffic that is extra full dump become fairly rare. The sequence amounts can be used to differentiate routes that are stale brand new ones and therefore steer clear of the development of loops [4]. Every mobile facility maintains a routing desk that lists all offered destinations, a number of hops to achieve the resort, therefore, the series quantity designated because of the location node. If you have room when you look at the progressive modify package then those records might be integrated whose sequence numbers has changed. a full dump delivers the entire routing table to the friends and might span numerous packages whereas in an incremental improve just those entries through the routing dining table are sent which includes a metric modification because the latest modify plus it must easily fit into a packet.

The Dynamic Source Routing protocol (DSR) is a simple and efficient routing protocol designed specifically for use in multi-hop wireless ad hoc networks of mobile nodes. DSR allows the network to be completely self-organizing and selfconfiguring, without the need for any existing network infrastructure or administration [5]. It is a reactive protocol and all aspects of the protocol operate entirely on-demand basis. It works on the concept of source routing. Source routing is a routing technique in which the sender of a packet determines the complete sequence of nodes through which, the packets are forwarded. In this routing the intermediate nodes do not need to maintain up to date routing information in order to route the packets they forward.

The protocol is composed of the two main mechanisms of "Route Discovery" and "Route Maintenance". DSR requires each node to maintain a route - cache of all known self - to destination pairs. If a node has a packet to send, it attempts to use this cache to deliver the packet. If the destination does not exist in the cache, then a route discovery phase is initiated to discover a route to destination, by sending a route request [6]. This request includes the destination address, source address and a unique identification number.

If a route is available from the route -cache, but is not valid any more, a route maintenance procedure may be initiated. A node processes the route request packet only if it has not previously processes the packet and its address is not present in the route cache. A route reply is generated by the destination or by any of the intermediate nodes when it knows about how to reach the destination.

Wireless Routing Protocol Basics:-There are several unicast routing algorithms that have been developed for MANETs that have their own unique characteristic strengths and weaknesses. We do describe in detail, however, all protocols that we felt were relevant to this work [7]. Different algorithms may have benefits in different topologies and motion scenarios and for different application scales.

The simplest wireless routing protocol is called flooding and as the name implies, a message is sent by a node to all its neighbors who send it out to all their neighbors and so on until it reaches the desired destination. This is one method known to guarantee delivery of packets provided at least one path exists between any two nodes. It has a great drawback, however, in that it wastes a lot of the limited bandwidth available, and if all nodes were to flood all other nodes, there would be too much interference, causing what is known as the Broadcast Storm problem [9].

Classification of Routing Protocols:-Most protocols can be classified in several ways. Some are classified as reactive or on- demand while others are proactive. In general, a proactive protocol finds routes in advance while a reactive protocol finds routes to the destination only when it absolutely must. For example, Ad hoc On demand Distance Vector routing (AODV) [11] is an on-demand protocol since no protocol information is transmitted before an application decides to send data and no data is sent until a route is formed, whereas Destination Sequenced Distance Vector protocol (DSDV) [14] is a more proactive protocol in which routes are discovered and stored even before they are needed.

Proactive protocols generally generate much more traffic than on-demand protocols. A third general category is a hybrid algorithm that effectively combines multiple characteristics in a unique and meaningful way. The hybrid network is an extension of a structured network, in which a mobile number may hook up to an accessibility point (AP) making use of multi-hop wireless routes via various another mobile hosts. The APs include a configuration to use a single or several readily available networks. Mobile host and wireless routers can identify their operating stations 
dynamically through station switching hybrid wireless networks (for example., multi hop cellular systems) has been shown to be a far better network design for the generation that is next networking sites. It will also help to deal with the strict end to end QoS specifications of various solutions. Hybrid networks synergistically blend system channels and MANETs to leverage each other.

For instance it integrates a mobile wireless Ad Hoc Network (MANET) and infrastructure that is wireless showed a better approach subsequent generation wireless companies. Hybrid wireless channels (for example., multihop cellular networks) have now been proven to be an improved network structure for the following generation wireless systems and can help deal with the stringent end-toend QoS criteria of various solutions. Hybrid channels infrastructure that synergistically combines and MANETs to leverage each other. Especially, infrastructure networking sites improve the scalability of MANETs, while MANETs instantly establish self-arranging networks, extending the insurance coverage regarding the system channels [15]. In a vehicle opportunistic access system (a case of hybrid companies), folks in automobiles have to publish or install video from remote Internet machines through accessibility guidelines (APs) (for example. base stations) spreading call at an urban area.

Since it is not likely that the bottom programs include the entire town to keep sufficiently strong transmission everywhere to guide a credit card application demanding higher website link costs, the vehicles themselves can develop a MANET to increase the coverage regarding the base station, providing steady circle relationships.

\section{Proposed Methodology}

Security optimization with the help of the k-copy cloud based protocol

Our routing algorithm can be depicted as follows,

1. Deploy a system of $\mathrm{N}$ hubs put arbitrarily in a zone of $\mathrm{x}$ Y sq. meters

2. Select any source (S) and goal (D) from the system for directing procedure

3. Let the euclidean separation between hub $\mathrm{S}$ and $\mathrm{D}$ be dref
4. Select all hubs from the system, where the accompanying conditions are fulfilled,

o dsn + dnd $>$ dref

o dsn $<$ dref

o dnd $<$ dref

where, $\mathrm{dsn}=$ Distance between source to chosen hub

$\mathrm{dnd}=$ Distance between chosen hub to goal

1. This channels in just those hubs which are in the steering way, and evacuates every single other hub

2. For every hub in the way, assess the accompanying measurement,

$$
\text { Metric }=\mathrm{di} / \mathrm{Ei}
$$

where, $\mathrm{di}=$ Distance between the hubs

$\mathrm{Ei}=$ Energy of the source hub

1. Start the hub choice from the source till the goal hub is come to. Once came to, send the information on the chose way

2. Before sending the information, apply information accumulation at the source hub

3. Split the amassed information into $\mathrm{k}$ parts, where $\mathrm{k}$ is the quantity of channels accessible for directing

4. Send the information on all the $\mathrm{k}$ channels from the source hub to the goal

5. Repeat this procedure for all interchanges

The above calculation ensures that the information is sent from the source to goal with least postponement, and least vitality because of information total, multichannel correspondence and consolidation of $\mathrm{d} / \mathrm{E}$ factor in the steering procedure. The throughput is upgraded also because of progress in postponement and diminished parcel misfortune due to multichannel correspondence. This ensures the parcel is transmitted in the practically same planning interim as the past bundles, consequently decreasing the jitter of the system. The detailed result analysis is mentioned in the next section.

\section{Results and Analysis}

We simulated our routing protocol in the network simulator version 2.34 environment, under the following network conditions,

Table 1. Network Parameters.

\begin{tabular}{ll}
\hline Network parameter & Value \\
\hline Network type & Wireless \\
Number of nodes & 30 to 100 \\
Network area & $300 \mathrm{~m} \times 300 \mathrm{~m}$ \\
Routing protocols & QoS aware \\
Packet size & 1000 bits @ 0.001 packets per second \\
Number of communications & 2 to 20 \\
Initial node energies & Randomized, with maximum energy of $1000 \mathrm{~mJ}$ per node \\
& $2 \mathrm{~mJ}$ per transmission \\
Energy model & $1 \mathrm{~mJ}$ per reception \\
& $0.1 \mathrm{~mJ}$ idle energy \\
\hline
\end{tabular}

We compared our proposed protocol with the AODV routing for the wireless network, and the following parameters were obtained. 
Table 2. Performance Comparison of Delay parameter.

\begin{tabular}{|c|c|c|c|c|c|c|}
\hline Nodes & Communications & Delay AODV (ms) & Delay Proposed (ms) & $\begin{array}{l}\text { Delay Proposed } \\
\text { with Cloud (ms) }\end{array}$ & $\begin{array}{l}\text { \% Improvement } \\
\text { Proposed }\end{array}$ & $\begin{array}{l}\text { \% Improvement } \\
\text { Proposed with Cloud }\end{array}$ \\
\hline 20 & 2 & 0.31 & 0.24 & 0.21 & 21.74 & 32.26 \\
\hline 20 & 3 & 0.35 & 0.27 & 0.23 & 22.78 & 34.29 \\
\hline 20 & 4 & 0.38 & 0.26 & 0.24 & 31.1 & 36.84 \\
\hline 20 & 5 & 0.41 & 0.33 & 0.3 & 18.45 & 26.83 \\
\hline 20 & 6 & 0.44 & 0.32 & 0.31 & 27.39 & 29.55 \\
\hline 20 & 7 & 0.45 & 0.35 & 0.32 & 22.17 & 28.89 \\
\hline 20 & 8 & 0.48 & 0.36 & 0.34 & 24.85 & 29.17 \\
\hline 20 & 9 & 0.49 & 0.33 & 0.35 & 31.65 & 28.57 \\
\hline 20 & 10 & 0.56 & 0.45 & 0.4 & 19.47 & 28.57 \\
\hline 50 & 5 & 0.37 & 0.32 & 0.28 & 12.49 & 24.32 \\
\hline 50 & 6 & 0.39 & 0.28 & 0.29 & 27.31 & 25.64 \\
\hline 50 & 8 & 0.52 & 0.41 & 0.34 & 20.32 & 34.62 \\
\hline 50 & 12 & 0.63 & 0.49 & 0.38 & 23 & 39.68 \\
\hline 50 & 15 & 0.65 & 0.5 & 0.42 & 22.83 & 35.38 \\
\hline 50 & 20 & 0.73 & 0.52 & 0.46 & 28.61 & 36.99 \\
\hline 75 & 5 & 0.66 & 0.46 & 0.39 & 30.32 & 40.91 \\
\hline 75 & 10 & 0.69 & 0.54 & 0.47 & 22.64 & 31.88 \\
\hline 75 & 15 & 0.72 & 0.53 & 0.49 & 26.16 & 31.94 \\
\hline 75 & 20 & 0.79 & 0.55 & 0.51 & 29.92 & 35.44 \\
\hline 100 & 5 & 0.46 & 0.32 & 0.28 & 30.39 & 39.13 \\
\hline 100 & 10 & 0.7 & 0.47 & 0.4 & 32.43 & 42.86 \\
\hline 100 & 15 & 0.79 & 0.59 & 0.49 & 26.18 & 37.97 \\
\hline 100 & 20 & 0.82 & 0.60 & 0.51 & 27.2 & 37.8 \\
\hline \multicolumn{2}{|c|}{ Mean Improvement } & 0.55 & 0.412 & 0.3656 & $26 \%$ & 34.24 \\
\hline
\end{tabular}

Similar comparisons were made for energy, packet delivery ratio, throughput and jitter. The following table shows the performance comparison for all the 5 parameters,

Table 3. Performance comparison for all the 5 parameters.

\begin{tabular}{llllll}
\hline Parameter & AODV & Proposed & Proposed with Cloud & \% Improvement & \%Improvement with Cloud \\
\hline Average Delay (ms) & 0.556 & 0.412 & 0.351 & $26 \%$ & $36.87 \%$ \\
Average Energy (mj) & 3.126 & 2.198 & 1.963 & $29 \%$ & $37.2 \%$ \\
Average PDR(\%) & 99.5 & 98.6 & 99.2 & $0 \%$ & $0.3 \%$ \\
Average Throughput(kbps) & 137.8 & 134.9 & 136.7 & $-0.50 \%$ & $0.8 \%$ \\
Average Jitter (ms) & 0.0062 & 0.0058 & 0.006 & $7 \%$ & $3.23 \%$ \\
\hline
\end{tabular}

From the above table we can see that the system delay has been limited (also can be shown from the graph), the vitality utilization has been decreased by keeping up a consistent normal bundle conveyance proportion and normal throughput.

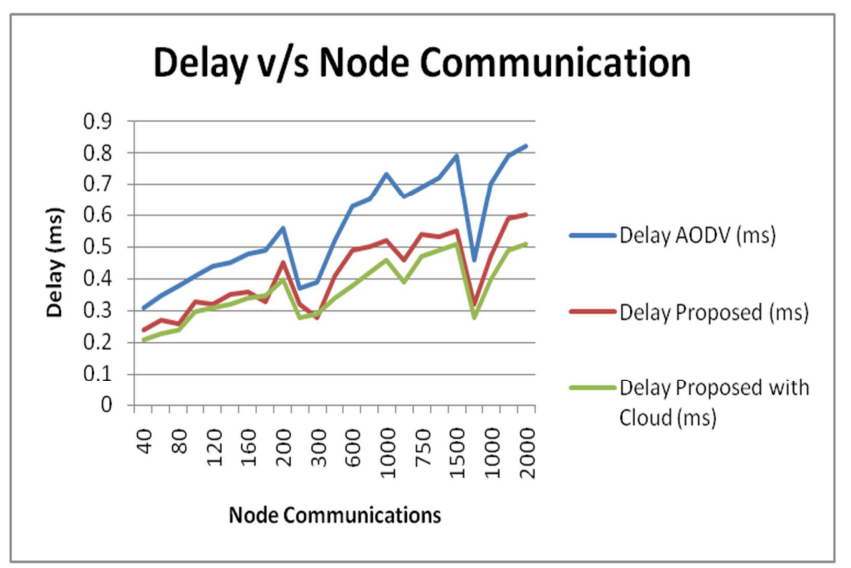

Figure 1. Delay $v / s$ Number of communications.

The general parcel conveyance jitter has likewise been marginally improved utilizing the AI approach. The postponement is decreased because of determination of least separation hubs for steering, while vitality is diminished in view of its consideration in the directing measurement as a conversely relative parameter. Because of decrease in postponement, the jitter is likewise diminished and along these lines it makes the system progressively dependable and predictable as far as parcel conveyance times at the collector. The PDR and throughput of AODV is as of now enhanced, and hence there in insignificant extent of progress around there. Also the security aspects of the research are improved with the help of the k-copy scheme as the data is more securely visible from source to destination without being compromised in the route.

The improvement of the parameters can also be visualized from the following graph,

As we can see all parameters are either optimized or are almost similar as compared to the existing standard protocol. We prescribe scientists to additionally assess this AI directing system so as to check it's feasibility for the applications for which they would plan the correspondence arrange. 


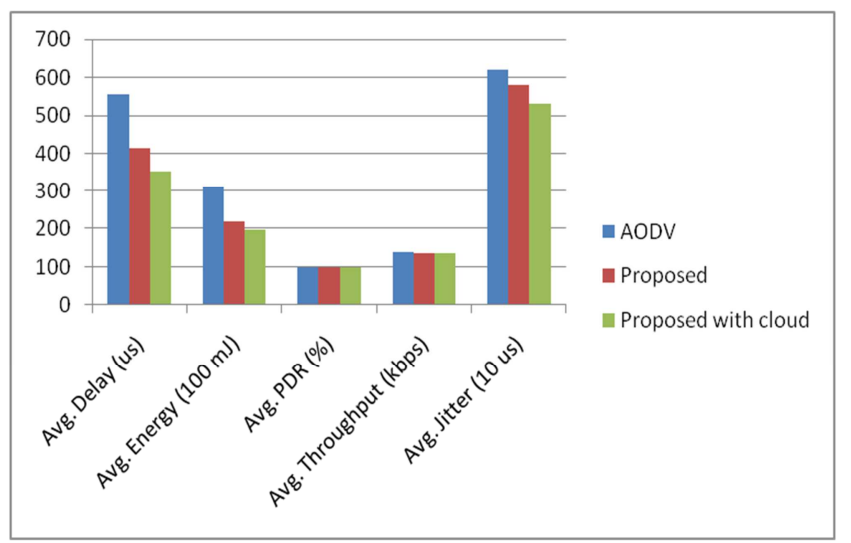

Figure 2. Overall performance of parameters.

\section{Conclusion}

Here we use a Quality of Service (QoS) aware routing protocol which combines delay \& energy aware routing with data aggregation \& multichannel communication in order to reduce the energy consumption, reduce the end to end delay and improve the network throughput by offloading the computational issues to the cloud and get back results from it. This increases the energy \& delay needed for communication between the cloud and network marginally, which is compensated by the computational efficiency of the cloud.

The proposed approach when applied to the wireless network gives a significant improvement in network performance, when compared with the recent de-facto AODV routing algorithm. The performance improvement to network lifetime is more than $25 \%$, while the delay minimization is more than $20 \%$ for a wide variety of network simulation parameters.

\section{Future Work}

As a future work, we plan to realize the protocol using hardware implementation in a real time wireless based network, due to the low cost nature of Arduino based wireless nodes, the hardware realization can be done in a closed lab environment. We also intend to research more into the QOS improvement of the wireless networks by incorporating more parameters into our machine learning protocol, and also adding Q-Learning and deep nets into the routing algorithm, which can adapt to the network patterns and select the most optimum route intelligently and in real time, with minimum on-the-fly complexity.

\section{References}

[1] [Supriya Bamane et al., 2013] Supriya Bamane, Rajesh Singh "AODV Based Improved Method for Detecting Good Neighbor Nodes" in International Journal of Emerging Technology and Advanced Engineering (ISSN 2250-2459, ISO 9001: 2008 Certified Journal), Volume 3, Issue 7, July2013.

[2] [Supriya Bamane et al., 2014] Supriya Bamane, Rajesh Singh
"Detecting Good Neighbor Nodes and Finding Reliable Routing Path Based on AODV Protocol", IOSR Journal of Computer Engineering (IOSR-JCE) e-ISSN: 2278-0661, pISSN: 2278-8727 Volume 16, Issue 1, Ver. I, pp 12-19, Jan. 2014.

[3] [Dr. S. S. Dhenakaran et al., 2013] Dr. S. S. Dhenakaran, A. parvarthavarthani "An Overview of Routing Protocols in Mobile Ad-Hoc Network " International Journal of Advanced Research in Computer Science and Software Engineering, ISSN: 2277 128X Volume 3, Issue 2, pp 251-259, February 2013.

[4] [Pawan Kumar Verma et al., 2010] Pawan Kumar Verma, Tarun Gupta, Nitin Rakesh, Nitin Nitin, “ A Mobile ad-Hoc Routing Algorithm with Comparative Study of Earlier Proposed Algorithms," International Journal of Scientific Reserch on Communications, Network and System Sciences, pp 289-293, 2010.

[5] [Ze Li, Haiying Shen et al., 2014] Ze Li, Haiying Shen,” A QoS-Oriented Distributed Routing Protocol for Hybrid Wireless Networks", IEEE Transaction on Mobile Computing, pp 693-708, Vol 13, No. 3, March 2014.

[6] [Shunyuan Yey et al., 2012] Shunyuan Yey, Yanming Shenz, Shivendra Panwar," An O (1) Scheduling Algorithm for Variable-size Packet Switching Systems" The 8th International Conference on Informatics and Systems, pp 4045, 14-16 May 2012.

[7] [Jasmine Norman et al., 2013] Jasmine Norman," Optimized Routing for Sensor Networks using Wireless Broadcast Advantage" Wseas Transaction on Information Science and Applications, E-ISSN: 2224-3402, Issue 5, Volume 10, May 2013.

[8] [Netrali Patil et al., 2014] Netrali Patil, Prof. Suraj Patil, Prof. Prashant Mishra, Prof. Sachin Chavan," AODV Based Improved Method for Detecting Good Neighbour Nodes with Energy Efficiency" International Journal of Application or Innovation in Engineering \& Management (IJAIEM), ISSN 2319 - 4847, Volume 3, Issue 4, April 2014.

[9] [Vinay. P. Viradia et al., 2003] Vinay. P. Viradia," Improved AODV Routing Protocol for MANET", Int. Journal of Engineering Research and Applications, ISSN: 2248-9622, Vol. 4, Issue 1 (Version 3), pp. 378, 2003.

[10] [Krishna Gorantala et al., 2006] Krishna Gorantala, "Routing Protocols in Mobile Ad-hoc Networks", Proceedings of International Conference on Open Source Computing INCOSC-08, A Master thesis in computer science, pp 1-36, 2006.

[11] [S. Ibrahim et al., 2008] S. Ibrahim, K. Sadek, W. Su and R. Liu, "Cooperative Communications with Relay Selection: When to cooperate and Whom to Cooperate with?," IEEE Transaction on Wireless Communication, vol. 7, no. 7, pp 2814-2827, July 2008.

[12] [Elizabeth M. Royer et al., 1999] Elizabeth M. Royer "A Review of Current Routing Protocols for Ad Hoc Mobile Wireless Networks" University of California, Santa Barbara Chai-Keong Toh, Georgia Institute of Technology, IEEE Personal Communications, pp 46-55, April 1999.

[13] [Silvia Giordano et al., 2002] Silvia Giordano, Ljubica Blazevic, Ivan Stojmenovic," Position Based Routing Algorithms For Ad Hoc Networks: A Taxonomy, 2002. 
[14] [Martin Mauve et al., 2001] Martin Mauve, Jörg Widmer, Hannes Hartenstein," A Survey on Position-Based Routing in Mobile Ad-Hoc Networks (2001), IEEE Network, ISSN0890-8044, November/December 2001.

[15] [Young-Bae Ko et al., 1998] Young-Bae Ko, Nitin Vaidya,"Location-Aided Routing (LAR) in Mobile Ad Hoc Networks", ACM MOBICOMl 9S Dallas Texas USA, 1998.

[16] [Wen-Hwa Liao et al., 2001] Wen-Hwa Liao, Yu-Chee Tseng, Jang-Ping Sheu," GRID: A Fully Location Aware Routing Protocol for Mobile Ad hoc Networks", Telecommunication Systems, Telecommunication Systems 18: 1-3, 37-60, 2001.
[17] [Sze-Yao Ni et al., 1999] Sze-Yao Ni, Yu-Chee Tseng, YuhShyan Chen, and Jang-Ping Sheu," The Broadcast Storm Problem in a Mobile Ad Hoc Network", ACM Mobicom '99 Seattle Washington USA, 1999.

[18] [Robert S. Gray et al., 2000] Robert S. Gray," Soldiers, Agents and Wireless Networks: A Report on a Military Application", PAAM 2000 in Manchester, England, 2000. 